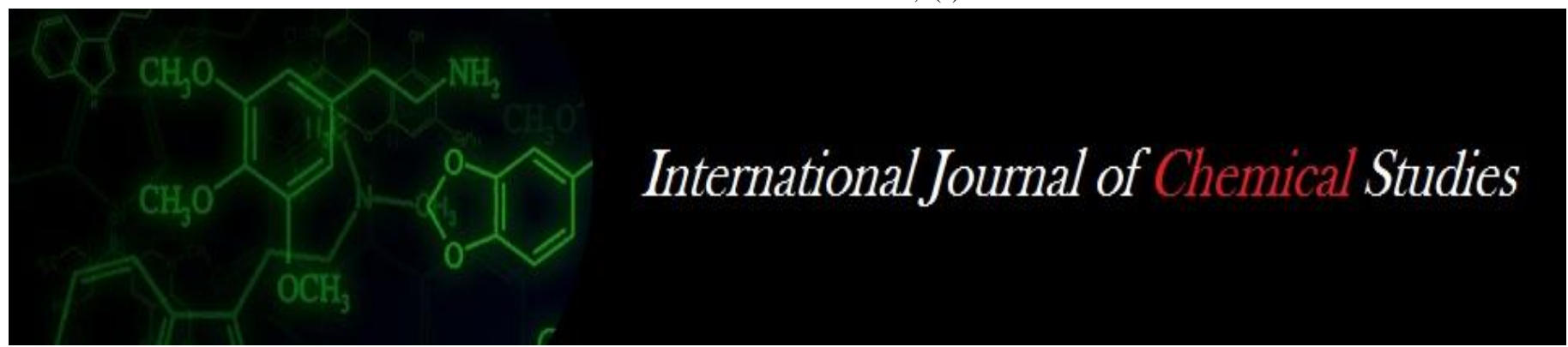

P-ISSN: 2349-8528

E-ISSN: 2321-4902

www.chemijournal.com

IJCS 2020; 8(4): 2652-2655

(C) 2020 IJCS

Received: 18-05-2020

Accepted: 22-06-2020

\section{Rukhsana Jan}

Division of Agronomy, Sher-e-

Kashmir University of

Agricultural Sciences and

Technology of Kashmir,

Shalimar Srinagar, Jammu and

Kashmir, India

\section{Khalid UI Islam Rather}

Division of Statistics and

Computer Science, Sher-e-

Kashmir University of

Agricultural Sciences and

Technology of Jammu, Chatha

Jammu, Jammu and Kashmir,

India

\section{Rizwan Yousuf}

Division of Statistics and

Computer Science, Sher-e-

Kashmir University of

Agricultural Sciences and

Technology of Jammu, Chatha

Jammu, Jammu and Kashmir,

India
Corresponding Author:

Rukhsana Jan

Division of Agronomy, Sher-e-

Kashmir University of

Agricultural Sciences and

Technology of Kashmir,

Shalimar Srinagar, Jammu and

Kashmir, India

\section{Growth, lodging and yield of transplanted rice (Oryza sativa L.) under Kashmir conditions as influenced by nitrogen and silicon applications}

\author{
Rukhsana Jan, Khalid UI Islam Rather and Rizwan Yousuf
}

DOI: https://doi.org/10.22271/chemi.2020.v8.i4ae.10041

\begin{abstract}
The field experiment entitled "Growth, lodging and yield of transplanted rice (Oryza sativa L.) under Kashmir conditions as influenced by nitrogen and silicon applications was conducted at Agronomy Research Farm of Sher-e-Kashmir University of Agricultural Sciences and Technology of Kashmir during Kharif season 2014 and 2015. The soil of the experiment was silty clay loam in texture, neutral in reaction with medium available nitrogen $\left(442.88 \mathrm{~kg} \mathrm{ha}^{-1}\right)$, phosphorus $\left(9.3 \mathrm{~kg} \mathrm{ha}^{-1}\right)$, potassium $(221.60 \mathrm{~kg}$ $\left.\mathrm{ha}^{-1}\right)$ and low available silicon $\left(280.23 \mathrm{~kg} \mathrm{ha}^{-1}\right)$. The experiment was laid out in Factorial Randomized Completely Block Design assigning combinations of nitrogen levels and silicon applications with four replications. The results revealed that the growth character viz. dry matter accumulation was significantly highest with $180 \mathrm{kgN} \mathrm{ha}^{-1}$ from $30-45 \mathrm{DAT}$. But from 60DAT upto at harvest, dry matter accumulation was significantly highest with $120 \mathrm{kgN} \mathrm{ha}^{-1}$ during 2014 and 2015 . Culm thickness was significantly highest with $120 \mathrm{kgN} \mathrm{ha}^{-1}$ at all growth stages during both the years. Internodal length (except mid tillering) were significantly superior with $180 \mathrm{kgN} \mathrm{ha}^{-1}$ at all growth stages during both the years. The lodging parameters $v i z$. breaking resistance and bending moment were significantly highest with $120 \mathrm{kgN}$ $\mathrm{ha}^{-1}$ while lodging index decreased with the same treatment during both the years. Significantly highest grain yield $\left(75.61 \mathrm{q} \mathrm{ha}^{-1}\right.$ and $\left.76.39 \mathrm{q} \mathrm{ha}^{-1}\right)$ and straw yield $\left(93.38 \mathrm{q} \mathrm{ha}^{-1}\right.$ and $\left.94.56 \mathrm{q} \mathrm{ha} \mathrm{h}^{-1}\right)$ were recorded with $120 \mathrm{kgN} \mathrm{ha}^{-1}$ during both the years respectively. So far as application of silicon is concerned, $15 \% \mathrm{Si}$ caused significant improvement in crop growth parameters viz. dry matter accumulation, culm thickness and internode length during 2014 and 2015. Similar trend was also observed for grain yield $\left(76.41 \mathrm{q} \mathrm{ha} \mathrm{q}^{-1}\right.$ and $\left.77.11 \mathrm{q} \mathrm{ha}^{-1}\right)$ and straw yield $\left(94.36 \mathrm{q} \mathrm{ha}^{-1}\right.$ and $\left.95.09 \mathrm{q} \mathrm{ha}^{-1}\right)$ during both the years. The lodging parameters viz. breaking resistance and bending moment were significantly highest with $15 \% \mathrm{Si}$ while lodging index decreased with the same treatment during both the years.
\end{abstract}

Keywords: B:C ratio, growth, lodging, nitrogen, rice, silicon, yield

\section{Introduction}

Nitrogen fertilizer is an important practice for increasing rice yield. It is essential to the rice plant, with about 75 per cent of leaf nitrogen associated with chloroplasts, which are physiologically important in dry matter production (Dalling, 1995) ${ }^{[2]}$. The presence of nitrogen in excess promotes development of the above ground organs with abundant dark green (high chlorophyll) tissues of soft consistency and relatively poor root growth. This increases the risk of lodging and reduces the plant resistance to harsh climatic condition and foliar diseases (Mohammadin et al. 2011) ${ }^{[14]}$.

Silicon is usually considered as one of the most important beneficial elements for rice production as rice requires large amounts of silica for its growth. The silicon content of soils can vary dramatically from $<1$ to 45 per cent by dry weight basis (Sommer et al. 2006) ${ }^{[21]}$ and its uptake by plants takesplace in the form of silicic acid $\left[\mathrm{Si}(\mathrm{OH})_{4}\right]$. It is estimated that nearly $20 \mathrm{~kg}$ of silica is removed from the soil by rice plants for production of $100 \mathrm{~kg}$ brown rice (Dobermann and Fairhurst, 1997) ${ }^{[3]}$.

Lodging may occur in vigorously growing rice plants after heading stage. It constitutes a major constraint to rice production, especially in high yielding environments. It causes direct loss in grain yield and quality (Fallah, 2000) ${ }^{[4]}$. Silicon helps plants to overcome multiple stresses including biotic stresses such as insect-pests and diseases like blast, brown spot and sheath 
blight and also abiotic stresses such as metal toxicity, salinity, drought and temperature (Ma, 2004 and Liang et al. 2007) ${ }^{[13,}$

10]. Keeping in view the above facts, the present study entitled" Growth, lodging and yield of transplanted rice (Oryza sativa L.) under Kashmir conditions as influenced by nitrogen and silicon applications was designed with following objectives:

To study the impact of silicon and nitrogen on growth and yield of rice

To study the effect of nitrogen and silicon on lodging

\section{Material and Methods}

Field experiment was conducted at Research Farm of Sher-eKashmir University of Agricultural Sciences and Technology of Kashmir, Shalimar during Kharif season, 2014 and 2015. The factorial experiment (two factors) based on randomised complete block design with four replications was laid out. The factors included three $\mathrm{N}$ levels $\left(\mathrm{N}_{1}: 120, \mathrm{~N}_{2}: 150, \mathrm{~N}_{3}: 180\right.$ $\mathrm{kg} / \mathrm{ha}$ ) and four $\mathrm{Si}$ applications $\left(\mathrm{Si}_{0}\right.$ : Control, $\mathrm{Si}_{1}: 5 \%, \mathrm{Si}_{2}$ : $10 \%$ and $\left.\mathrm{Si}_{3}: 15 \%\right)$ and the treatment combinations were $\mathrm{N}_{1} \mathrm{Si}_{0}, \mathrm{~N}_{1} \mathrm{Si}_{1}, \mathrm{~N}_{1} \mathrm{Si}_{2}, \mathrm{~N}_{1} \mathrm{Si}_{3}, \mathrm{~N}_{2} \mathrm{Si}_{0}, \mathrm{~N}_{2} \mathrm{Si}_{1}, \mathrm{~N}_{2} \mathrm{Si}_{2}, \mathrm{~N}_{2} \mathrm{Si}_{3}, \mathrm{~N}_{3} \mathrm{Si}_{0}$, $\mathrm{N}_{3} \mathrm{Si}_{1}, \mathrm{~N}_{3} \mathrm{Si}_{2}$ and $\mathrm{N}_{3} \mathrm{Si}_{3}$. The various growth, lodging, yield parameters and relative economics were recorded. Culm thickness was recorded at 15 days interval after transplanting till harvest using veirneir clipper. Breaking resistance can be measured with a bending hardness tester. Bending moment (g.cm) using the formula,

$\mathrm{BMN}_{3}=$ Length from the lowest node of $\mathrm{N}_{3}$ to the top of panicle $\times\left(\mathrm{W}_{1}+\mathrm{W}_{2}\right)$

Where $\mathrm{w}_{1}$ is the fresh weight of whole plant and $\mathrm{w}_{2}$ is the fresh weight of third internode with leaf sheath. Lodging index $(\%)=$ Bending moment/Breaking resistance $\times 100$.

\section{Result and Discussion}

Data from table 1 showed an increase in dry matter accumulation with the advancement of crop. Application of $180 \mathrm{kgN} \mathrm{ha}^{-1}$ recorded significantly highest crop dry matter accumulation from 30DAT to 45DAT during 2014 and 2015. There are many reports on the increased of total dry matter due to increased nitrogen fertilizer application (Prasad, 1981 and Park, 1987) ${ }^{[18,15]}$. But from 60 DAT upto at harvest, $120 \mathrm{kgN} \mathrm{ha}^{-1}$ recorded significantly highest crop dry matter accumulation followed by $150 \mathrm{kgN} \mathrm{ha}^{-1}$ during both the consecutive years. It is because of increased number of tillers thereby increased dry matter production. Similar results were also reported by Stalin et al. (1999) ${ }^{[22]}$, Rao et al. (2004) ${ }^{[19]}$ and Prasad et al. $(2011)^{[17]}$.

With regards to the effect of silicon applications, $15 \% \mathrm{Si}$ recorded significantly highest dry matter accumulation followed by $10 \% \mathrm{Si}$ which remained statistically at par with $5 \% \mathrm{Si}$. It might be the maintenance of photosynthetic activity due to silicon fertilization could be one of the reason for the increased dry matter production (Agurie et al. 1992) ${ }^{[1]}$. Culm thickness is important character to express the lodging index of the plant, which ultimately influences the economic yield. Data pertaining to culm thickness in table 2 indicated that significantly highest culm thickness was recorded with $120 \mathrm{kgN} \mathrm{ha}^{-1}$ followed by $150 \mathrm{kgN} \mathrm{ha}^{-1}$ from $30 \mathrm{DAT}$ upto at harvest during both the years. Reason is being that optimum nitrogen level viz. $120 \mathrm{kgN} \mathrm{ha}^{-1}$ helps in stem strength and higher nitrogen fertilizer levels primarily induced significant reduction of dry weight which resulted in lower breaking resistance and consequently, reduced stem physical strength which caused a reduction in culm thickness thereby, resulted in poor lodging resistance of rice plants. Similar findings were reported by Wei et al. 2008; Yang et al. 2009; Wang et al. 2012 and Zhang et al. $2013^{[28,25,26,29]}$.

The data further revealed that among silicon applications, $15 \%$ Si recorded significantly higher culm thickness followed by $10 \% \mathrm{Si}$ remained at par with $5 \% \mathrm{Si}$ from $45 \mathrm{DAT}$ upto at harvest during both the consecutive years. It might be due to silicon improved epidermal cell wall thickness and size of vascular bundles which resulted in increases culm thickness. Fallah (2012) ${ }^{[5]}$ were also studied the same results. Data presented in table 3 indicated that internode length was significantly highest with $180 \mathrm{kgN} \mathrm{ha}^{-1}$ followed by $150 \mathrm{kgN}$ $\mathrm{ha}^{-1}$ at all growth stages. It might be due to higher plant height.

Among silicon applications, $15 \% \mathrm{Si}$ observed significantly highest internode length followed by $10 \% \mathrm{Si}$ being remained at par with $5 \% \mathrm{Si}$ at all growth stages except mid tillering during both the years. Data presented in table 4 indicated that breaking resistance and bending moment were significantly highest with $120 \mathrm{kgN} \mathrm{ha}^{-1}$ followed by $150 \mathrm{kgN} \mathrm{ha}^{-1}$ during both the years. It may be due to lower plant height and internode length. Guo et al. $2003^{[6]}$ were reported the similar results. It was further observed that among different silicon applications, $15 \% \mathrm{Si}$ recorded highest breaking resistance and bending moment followed by $10 \% \mathrm{Si}$ which was statistically at par with $5 \% \mathrm{Si}$ during both the consecutive years. It may be due to the thickening of cell wall of the sclerenchyma tissue in the culm and/or shortening and thickening of internodes or increase in silicon content of lower internodes provides mechanical strength to enable plant to resist lodging and also this study showed that breaking resistance and bending moment increased with increasing silicon concentration. Lee (1990) ${ }^{[9]}$; Liang (1994) ${ }^{[11]}$ and Takahashi (1995) ${ }^{[23]}$ were also noticed an increase in resistance to lodging due to application of silicon fertilizer to rice.

Out of the three nitrogen levels presented in table 4, application of $120 \mathrm{kgN} \mathrm{ha}^{-1}$ proved significantly efficient in controlling lodging followed by $150 \mathrm{kgN} \mathrm{ha}{ }^{-1}$ during both the years. This is in accordance to the study of Hui et al. (2013) ${ }^{[7]}$ where high nitrogen input increased basal internode length, breaking resistance and lodging index.

Among silicon applications, $15 \%$ Si proved significantly better measure for recording lodging index as compared to $10 \% \mathrm{Si}$ and $5 \% \mathrm{Si}$ during both the years. It could be ascribed to the fact that increased silicon content plant has been reported to increase mechanical strength of plant tissue, which resulted in reduced lodging index (Takahashi et al. 1990 and Liang 1994) $[24,11]$. Shimoyama $(1958)^{[20]}$ has mentioned that thickness of culm walls and vascular bundles becomes larger when silicon is applied. Silica deposited in these plant sections also contributes to an increase in the mechanical strength of culms. Therefore, sufficient supply of silicon has an effect on the stability of culms and serves to decrease the risk of lodging. From the table 5 indicated that $120 \mathrm{kgN} \mathrm{ha}^{-1}$ recorded significantly higher grain yield followed by $150 \mathrm{kgN} \mathrm{ha}^{-1}$ during both the years. It is because of highest yield attributing characters which ultimately caused the increase of rice grain yield. Similar results were reported by Huang et al. $2011^{[8]}$. Also, the increment of grain yield by the application of nitrogen upto a certain level was reported by Zhaowen et al. $2013^{[30]}$ Among different silicon applications, 15\% Si recorded the highest grain yield followed by $10 \% \mathrm{Si}$ being at par with $5 \%$ Si during both the years.

It is because of silicon is responsible to control stomatal activity, photosynthesis, water use efficiency which ultimately results in better vegetative growth results in higher grain 
yield. Data presented in table 5 indicated that $120 \mathrm{kgN} \mathrm{ha}^{-1}$ provided significantly higher straw yield.It might be due to the more number of dry matter accumulation which ultimately confirmed the greater yield of straw. Similar trend of the effect of nitrogen levels on straw yield were also reported by Pramanik and Bera (2013) ${ }^{[16]}$. Among different silicon applications, $15 \% \mathrm{Si}$ produced significantly highest straw yield during both the years. It may be attributed to leaf erectness which facilitated better penetration of sunlight leading to higher photosynthetic activity of plant and higher production of carbohydrates. Similar results were also noticed by Ma et al. $(1989)^{[12]}$.

Table 1: Impact of nitrogen and silicon on dry matter accumulation $\left(\mathrm{q} \mathrm{ha} \mathrm{h}^{-1}\right)$ of transplanted rice

\begin{tabular}{|c|c|c|c|c|c|c|c|c|c|c|}
\hline Years & 2014 & 5 & 5 & 2015 & 2014 & 2015 & 2014 & 015 & 2014 & 2015 \\
\hline reatments & 15D & AT & 301 & DAT & $45 \mathrm{I}$ & AT & $60 \mathrm{I}$ & DAT & $75 \mathrm{I}$ & DAT \\
\hline \multicolumn{11}{|c|}{ Nitrogen levels $\left(\mathrm{kg} \mathrm{ha}^{-1}\right)$} \\
\hline 120 & 0.91 & 5 & 12.98 & 13.84 & 35.28 & 37.16 & 60 & 66205 & 0.3 & \\
\hline $150\left(\mathrm{~N}_{2}\right)$ & 4.06 & & 13.26 & 14 & 35.64 & 37 & 58.92 & 6 & 4.5 & 26 \\
\hline $180\left(\mathrm{~N}_{3}\right)$ & 4.06 & 4.30 & 13.53 & 14.62 & 36.00 & 38.52 & 57.24 & 58.83 & 22.39 & 4.01 \\
\hline SEm \pm & 0.12 & 0.13 & 0.08 & 0.11 & 0.09 & 0.12 & 0.52 & 0.53 & 0.65 & 0.75 \\
\hline$\overline{\mathrm{D}(p \leq}$ & NS & NS & 0.24 & 0.34 & 0.28 & 0.37 & 1.53 & 1.57 & 1.92 & 2.21 \\
\hline \multicolumn{11}{|c|}{ Silicon applications (\%) } \\
\hline ntrol & 3.79 & & 13.11 & & 34.20 & & 5520 & & 0133 & 92.42 \\
\hline $5(S$ & 3.97 & 4.14 & 13.2 & 14. & 35.93 & 37.91 & 58.48 & 361.0 & 94.0 & 95.6 \\
\hline 10( & 4.10 & 4.27 & 13.31 & 14.27 & 36.03 & 38.06 & 59.83 & 62.38 & 95.3 & 97.0 \\
\hline 15( & 4.18 & 4.35 & 13.41 & 14.43 & 36.40 & 38.55 & 62.44 & 64.20 & 97.6 & 100.0 \\
\hline $\mathrm{SE}$ & 0.14 & 0.15 & 0.09 & 0.13 & 0.11 & 0.14 & 0.60 & 0.61 & 0.76 & 0.87 \\
\hline $\mathrm{D}(p \leq 0.05)$ & 0.42 & NS & NS & NS & 0.32 & 0.42 & 1.76 & 1.81 & 2.23 & 2.55 \\
\hline
\end{tabular}

Table 1: Contd.

\begin{tabular}{|c|c|c|c|c|c|c|c|c|}
\hline Years & $\mathbf{2 0 1 4}$ & $\mathbf{2 0 1 5}$ & $\mathbf{2 0 1 4}$ & $\mathbf{2 0 1 5}$ & $\mathbf{2 0 1 4}$ & $\mathbf{2 0 1 5}$ & $\mathbf{2 0 1 4}$ & $\mathbf{2 0 1 5}$ \\
\hline Treatments & 90 DAT & 105 DAT & 120 DAT & At harvest \\
\hline \multicolumn{8}{|c|}{ Nitrogen levels $\left(\mathbf{k g ~ h a}^{-1}\right)$} \\
\hline $120\left(\mathrm{~N}_{1}\right)$ & 149.50 & 152.45 & 159.81 & 162.62 & 164.60 & 167.13 & 168.37 & 170.65 \\
\hline $150\left(\mathrm{~N}_{2}\right)$ & 147.04 & 149.84 & 157.06 & 159.82 & 161.68 & 164.00 & 164.40 & 166.29 \\
\hline $180\left(\mathrm{~N}_{3}\right)$ & 144.86 & 145.60 & 150.55 & 153.48 & 153.94 & 156.54 & 156.60 & 157.97 \\
\hline $\mathrm{SEm} \pm$ & 0.71 & 0.74 & 0.93 & 0.95 & 0.97 & 0.99 & 1.19 & 1.06 \\
\hline $\mathrm{CD}(p \leq 0.05)$ & 2.08 & 2.17 & 2.72 & 2.79 & 2.86 & 2.92 & 3.49 & 3.13 \\
\hline \multicolumn{8}{|c|}{ Silicon applications (\%) } \\
\hline Control $\left(\mathrm{Si}_{1}\right)$ & 140.32 & 142.27 & 148.41 & 151.15 & 150.76 & 153.44 & 151.27 & 154.05 \\
\hline $5\left(\mathrm{Si}_{1}\right)$ & 147.39 & 149.61 & 155.18 & 158.81 & 160.89 & 162.45 & 164.06 & 165.41 \\
\hline $10\left(\mathrm{Si}_{2}\right)$ & 148.93 & 151.05 & 157.54 & 160.23 & 162.25 & 164.90 & 166.43 & 168.11 \\
\hline $15\left(\mathrm{Si}_{3}\right)$ & 151.90 & 154.25 & 162.10 & 164.38 & 166.39 & 169.44 & 170.74 & 172.32 \\
\hline $\mathrm{SEm} \pm$ & 0.82 & 0.85 & 1.07 & 1.09 & 1.12 & 1.15 & 1.37 & 1.23 \\
\hline $\mathrm{CD}(p \leq 0.05)$ & 2.41 & 2.51 & 3.15 & 3.22 & 3.30 & 3.38 & 4.04 & 3.61 \\
\hline
\end{tabular}

Table 2: Impact of nitrogen and silicon on culm thickness (mm) of transplanted rice

\begin{tabular}{|c|c|c|c|c|c|c|c|c|c|c|}
\hline Years & \multicolumn{6}{|c|}{\begin{tabular}{|l|l|l|l|l|}
2015 & 20142015 & 2014 & 2015
\end{tabular}} & \multicolumn{2}{|c|}{20142015} & \multicolumn{2}{|c|}{\begin{tabular}{|l|l|}
2014 & 2015 \\
\end{tabular}} \\
\hline Treatments & \multicolumn{2}{|c|}{ 15DAT } & \multicolumn{2}{|c|}{ 30DAT } & \multicolumn{2}{|c|}{ 45DAT } & \multicolumn{2}{|c|}{ 60DAT } & \multicolumn{2}{|c|}{ 75DAT } \\
\hline \multicolumn{11}{|c|}{ Nitrogen levels $\left(\mathrm{kg} \mathrm{ha}^{-1}\right)$} \\
\hline $120\left(\mathrm{~N}_{1}\right)$ & 0.35 & 0.37 & 1.74 & 1.80 & 10.61 & 11.86 & 17.19 & 18.35 & 19.80 & 20.91 \\
\hline $150\left(\mathrm{~N}_{2}\right)$ & 0.35 & 0.35 & 1.61 & 1.66 & 10.24 & 11.48 & 17.00 & 1 & 19.52 & 20.64 \\
\hline $180\left(\mathrm{~N}_{3}\right)$ & 0.33 & 0.35 & 1.45 & 1.52 & 9.88 & 11.06 & 16. & 17.85 & 19.17 & 20.27 \\
\hline SEm \pm & 0.01 & 0.02 & 0.04 & 0.05 & 0.09 & 0.10 & 0.05 & 0.06 & 0.08 & 0.08 \\
\hline $\mathrm{CD}(p \leq 0.05)$ & NS & NS & 0.13 & 0.14 & 0.26 & 0.30 & 0.17 & 0.19 & 0.24 & 0.26 \\
\hline \multicolumn{11}{|c|}{ Silicon applications (\%) } \\
\hline ontrol (Sio & 0.31 & 0.32 & 1.56 & 1.54 & 9.41 & 10.57 & 16.4 & 17.54 & 0. & 9.82 \\
\hline $5\left(\mathrm{Si}_{1}\right)$ & 0.33 & 0.35 & 1.57 & 1.65 & 10.15 & 4 & 110 & $\Omega$ & 19. & 0.56 \\
\hline $10\left(\mathrm{Si}_{2}\right)$ & 0.35 & 0.36 & 1.62 & 1.70 & 10.39 & 11 & 17. & 18.23 & 19.6 & 20.75 \\
\hline $15\left(\mathrm{Si}_{3}\right)$ & 0.37 & 0.39 & 1.65 & 1.74 & 11.02 & 12.26 & 17.49 & 18.61 & 20.19 & 21.31 \\
\hline SEm \pm & 0.02 & 0.02 & 0.05 & 0.05 & 0.10 & 0.11 & 0.06 & 0.07 & 0.09 & 0.10 \\
\hline $\mathrm{CD}(p \leq 0.05)$ & NS & NS & NS & NS & 0.30 & 0.34 & 0.19 & 0.22 & 0.28 & 0.30 \\
\hline
\end{tabular}

Table 2: Contd.

\begin{tabular}{|c|c|c|c|c|c|c|c|c|}
\hline Years & 2014 & $\mathbf{2 0 1 5}$ & $\mathbf{2 0 1 4}$ & $\mathbf{2 0 1 5}$ & $\mathbf{2 0 1 4}$ & $\mathbf{2 0 1 5}$ & $\mathbf{2 0 1 4}$ & $\mathbf{2 0 1 5}$ \\
\hline Treatments & 90DAT & 105DAT & 120DAT & At harvest \\
\hline \multicolumn{8}{|c|}{ Nitrogen levels (kg ha-1) } \\
\hline $120\left(\mathrm{~N}_{1}\right)$ & 20.85 & 21.64 & 21.78 & 22.89 & 21.90 & 23.13 & 22.14 & 23.23 \\
\hline $150\left(\mathrm{~N}_{2}\right)$ & 20.48 & 21.24 & 21.30 & 22.48 & 21.43 & 22.63 & 21.68 & 22.79 \\
\hline $180\left(\mathrm{~N}_{3}\right)$ & 20.01 & 20.77 & 20.90 & 22.01 & 21.02 & 22.15 & 21.13 & 22.23 \\
\hline $\mathrm{SEm} \pm$ & 0.12 & 0.13 & 0.13 & 0.14 & 0.14 & 0.16 & 0.15 & 0.14 \\
\hline $\mathrm{CD}(p \leq 0.05)$ & 0.37 & 0.39 & 0.39 & 0.41 & 0.41 & 0.48 & 0.44 & 0.42 \\
\hline \multicolumn{8}{|c|}{ Silicon applications $(\%)$} \\
\hline Control $\left(\mathrm{Si}_{0}\right)$ & 19.36 & 20.12 & 20.26 & 21.38 & 20.37 & 21.52 & 20.44 & 21.52 \\
\hline $5\left(\mathrm{Si}_{1}\right)$ & 20.44 & 21.21 & 21.38 & 22.53 & 21.50 & 22.72 & 21.80 & 22.88 \\
\hline $10\left(\mathrm{Si}_{2}\right)$ & 20.68 & 21.44 & 21.53 & 22.66 & 21.65 & 22.84 & 21.92 & 23.00 \\
\hline $15\left(\mathrm{Si}_{3}\right)$ & 21.30 & 22.09 & 22.15 & 23.29 & 22.27 & 23.46 & 22.45 & 23.59 \\
\hline $\mathrm{SEm} \pm$ & 0.14 & 0.15 & 0.15 & 0.16 & 0.16 & 0.19 & 0.17 & 0.18 \\
\hline $\mathrm{CD}(p \leq 0.05)$ & 0.43 & 0.45 & 0.46 & 0.48 & 0.48 & 0.58 & 0.51 & 0.54 \\
\hline
\end{tabular}

Table 3: Impact of nitrogen and silicon on internode length $(\mathrm{cm})$ of transplanted rice

\begin{tabular}{|c|c|c|c|c|c|c|c|c|}
\hline Years & $\mathbf{2 0 1 4}$ & $\mathbf{2 0 1 5}$ & $\mathbf{2 0 1 4}$ & $\mathbf{2 0 1 5}$ & $\mathbf{2 0 1 4}$ & $\mathbf{2 0 1 5}$ & $\mathbf{2 0 1 4}$ & $\mathbf{2 0 1 5}$ \\
\hline Treatments & Mid tillering & Panicle initiation & Anthesis & Maturity \\
\hline \multicolumn{8}{|c|}{ Nitrogen levels $\left(\right.$ kg ha $\left.^{-1}\right)$} \\
\hline $120\left(\mathrm{~N}_{1}\right)$ & 1.74 & 1.75 & 13.95 & 15.02 & 24.78 & 25.58 & 26.35 & 26.85 \\
\hline $150\left(\mathrm{~N}_{2}\right)$ & 1.76 & 1.78 & 14.56 & 15.62 & 25.80 & 27.19 & 27.39 & 28.66 \\
\hline $180\left(\mathrm{~N}_{3}\right)$ & 1.79 & 1.82 & 14.78 & 15.84 & 26.75 & 28.20 & 28.45 & 29.74 \\
\hline $\mathrm{SEm} \pm$ & 0.008 & 0.01 & 0.04 & 0.06 & 0.30 & 0.33 & 0.32 & 0.35 \\
\hline $\mathrm{CD}(p \leq 0.05)$ & 0.02 & 0.03 & 0.13 & 0.18 & 0.89 & 0.97 & 0.94 & 1.03 \\
\hline \multicolumn{8}{|c|}{ Silicon applications (\%) } \\
\hline Control $\left(\mathrm{Si}_{1}\right)$ & 1.74 & 1.76 & 14.04 & 15.02 & 24.01 & 24.88 & 25.17 & 26.19 \\
\hline $5\left(\mathrm{Si}_{1}\right)$ & 1.77 & 1.78 & 14.48 & 15.51 & 25.74 & 27.18 & 27.59 & 28.63 \\
\hline $10\left(\mathrm{Si}_{2}\right)$ & 1.77 & 1.79 & 14.51 & 15.60 & 26.12 & 27.37 & 27.85 & 28.81 \\
\hline $15\left(\mathrm{Si}_{3}\right)$ & 1.78 & 1.79 & 14.70 & 15.83 & 27.23 & 28.53 & 28.98 & 30.04 \\
\hline $\mathrm{SEm} \pm$ & 0.009 & 0.01 & 0.05 & 0.07 & 0.35 & 0.38 & 0.37 & 0.40 \\
\hline $\mathrm{CD}(p \leq 0.05)$ & $\mathrm{NS}$ & $\mathrm{NS}$ & 0.15 & 0.21 & 1.03 & 1.12 & 1.08 & 1.19 \\
\hline
\end{tabular}

Table 4: Impact of nitrogen and silicon on lodging parameters of transplanted rice

\begin{tabular}{|c|c|c|c|c|c|c|}
\hline Years & $\mathbf{2 0 1 4}$ & $\mathbf{2 0 1 5}$ & $\mathbf{2 0 1 4}$ & $\mathbf{2 0 1 5}$ & $\mathbf{2 0 1 4}$ & $\mathbf{2 0 1 5}$ \\
\hline Treatments & $\begin{array}{c}\text { Breaking resistance } \\
\text { (g. cm) }\end{array}$ & $\begin{array}{c}\text { Bending moment } \\
\text { (g.cm) }\end{array}$ & $\begin{array}{c}\text { Lodging index } \\
\text { (\%) })\end{array}$ \\
\hline \multicolumn{7}{|c|}{ Nitrogen levels $\left(\mathbf{k g ~ h a}^{-1}\right)$} \\
\hline $120\left(\mathrm{~N}_{1}\right)$ & 1659.93 & 1718.92 & 1589.98 & 1658.08 & 94.86 & 95.09 \\
\hline $150\left(\mathrm{~N}_{2}\right)$ & 1574.46 & 1644.83 & 1511.87 & 1586.34 & 95.89 & 96.06 \\
\hline $180\left(\mathrm{~N}_{3}\right)$ & 1485.84 & 1569.48 & 1438.26 & 1514.35 & 96.93 & 97.05 \\
\hline $\mathrm{SEm} \pm$ & 24.17 & 24.97 & 22.43 & 23.65 & 0.33 & 0.32 \\
\hline $\mathrm{CD}(p \leq 0.05)$ & 70.92 & 73.23 & 65.80 & 69.38 & 1.01 & 0.97 \\
\hline \multicolumn{7}{|c|}{ Silicon applications (\%) } \\
\hline Control $\left(\mathrm{Si}_{0}\right)$ & 1459.04 & 1531.27 & 1406.83 & 1476.71 & 97.80 & 97.92 \\
\hline $5\left(\mathrm{Si}_{1}\right)$ & 1544.55 & 1624.75 & 1490.16 & 1561.83 & 96.63 & 96.83 \\
\hline $10\left(\mathrm{Si}_{2}\right)$ & 1600.19 & 1668.03 & 1536.50 & 1611.32 & 96.28 & 96.62 \\
\hline $15\left(\mathrm{Si}_{3}\right)$ & 1689.87 & 1753.60 & 1619.99 & 1695.17 & 95.13 & 95.46 \\
\hline $\mathrm{SEm} \pm$ & 27.92 & 28.83 & 25.90 & 27.31 & 0.37 & 0.35 \\
\hline $\mathrm{CD}(p \leq 0.05)$ & 81.90 & 84.56 & 75.98 & 80.11 & 1.13 & 1.07 \\
\hline
\end{tabular}

Table 5: Impact of nitrogen and silicon on grain yield and straw yield of transplanted rice

\begin{tabular}{|c|c|c|c|c|}
\hline Years & 2014 & 2015 & 2014 & 2015 \\
\hline Treatments & & $\begin{array}{l}\text { Grain yield } \\
\left(\mathbf{q} \mathbf{h a}^{-1}\right)\end{array}$ & \multicolumn{2}{|c|}{$\begin{array}{l}\text { Straw yield } \\
\left(\mathbf{q} \mathbf{h a}^{-1}\right)\end{array}$} \\
\hline & \multicolumn{4}{|c|}{ Nitrogen levels $\left(\mathrm{kg} \mathrm{ha}^{-1}\right)$} \\
\hline $120\left(\mathrm{~N}_{1}\right)$ & 75.61 & 76.39 & 93.38 & 94.56 \\
\hline $150\left(\mathrm{~N}_{2}\right)$ & 73.55 & 74.52 & 91.23 & 92.09 \\
\hline $180\left(\mathrm{~N}_{3}\right)$ & 68.45 & 69.21 & 87.69 & 88.70 \\
\hline $\mathrm{SEm} \pm$ & 0.48 & 0.54 & 0.53 & 0.55 \\
\hline $\mathrm{CD}(p \leq 0.05)$ & 1.42 & 1.62 & 1.57 & 1.62 \\
\hline \multicolumn{5}{|c|}{ Silicon applications (\%) } \\
\hline Control $\left(\mathrm{Si}_{0}\right)$ & 66.87 & 67.67 & 84.55 & 86.49 \\
\hline $5\left(\mathrm{Si}_{1}\right)$ & 72.75 & 73.67 & 91.63 & 92.30 \\
\hline $10\left(\mathrm{Si}_{2}\right)$ & 74.12 & 75.04 & 92.52 & 93.26 \\
\hline $15\left(\mathrm{Si}_{3}\right)$ & 76.41 & 77.11 & 94.36 & 95.09 \\
\hline SEm \pm & 0.56 & 0.59 & 0.62 & 0.64 \\
\hline $\mathrm{CD}(p \leq 0.05)$ & 1.65 & 1.77 & 1.82 & 1.87 \\
\hline
\end{tabular}




\section{Conclusion}

The two year study revealed that silicon applications can significantly regulate plant growth, lodging and yield if applied at proper time with feasible concentration. As far as fertilization of rice crop is concerned-application of nitrogen fertilizer is an important practice for increasing rice yield. However, nitrogen applied in excess may limit yield due to lodging which promoted shading and susceptibility to insects and diseases. These effects could be minimized by the use of silicon. Considering the influence of nitrogen levels and silicon applications, it can be suggested that for realising economically higher grain yield of rice and reducing lodging with $120 \mathrm{~kg} \mathrm{ha}^{-1}$ with $15 \% \mathrm{Si}$ based nutrient management during 2014 and 2015 respectively.

\section{References}

1. Agurie S, Agara W, Kubota F, Kaufman PB. Physiological role of Silicon in photosynthesis and dry matter production in rice plants. Japan Journal of Crop Scince. 1992; 61:200-206.

2. Dalling MJ. The physiological basis of nitrogen redistributing during filling in cereals. P.55-71. In J. E. Harper et al. (ed.) Exploitation of physiological and genetic variability to enhance crop productivity. American Society of Plant Physiologists, Rockville, MD, 1995.

3. Dobermann A, Fairhurst T. Field Handbook. Nutritional disorders and nutrient management in rice. International Rice Research Institute (IRRI). Potash and phosphate Institute of Canada (PPIC), 1997, 162.

4. Fallah A. Effects of silicon and nitrogen on growth, lodging and spikelet filling in Rice (Oryza sativa L.). $\mathrm{Ph}$. Thesis. UPLB, 2000.

5. Fallah A. Silicon effect on lodging parameters of rice plants under hydroponic culture International Journal of Agriculture Science. 2012; 2(7):630-634.

6. Guo YH, Zhu SG, Zhang LB, Du H. Influence on the material characteristics of rice culms in different cultivation conditions. Journal of Shenyang Agricultural University. 2003; 34:4-7.

7. Hui LG, Hua ZX, Ka T, Rang HN, Feng PJ, Hui HT. Effect of nitrogen application on stem lodging resistance of rice and its morphological and mechanical mechanisms. Scientia Agriculture Sinica. 2013; 7:89-97.

8. Huang M, Zou YB, Jiang P, Bing XIA, Md I, Ao HJ et al. Relationship between Grain Yield and Yield Components in Super Hybrid Rice. Agricultural Sciences in China. 2011; 10:1537-1544.

9. Lee IJ. Effect of silicon application on grain ripening, hull formation as well as grain quality of rice. Research Reports of the Rural Development and Administration soil and Fertilizer. 1990; 32:15-23.

10. Liang Y, Sun W, Zhu Y, Christie P. Mechanisms of silicon mediated alleviation of abiotic stressin higher plants. A review Environmental Pollution. 2007; 147:422-428.

11. Liang YC. Effect of silicon application on rice grain yield. Plant nutrition of Soil Science. 1994; 25:22852297.

12. Ma JF, Nishimura K, Takahashi E. Effect of silicon on the growth of rice plant at different growth-stages. Soil Science and Plant Nutrition. 1989; 35:347-356.

13. Ma JF. Role of silicon in enhancing the resistance of plants to biotic abiotic stresses. Soil Science Plant Nutrition. 2004; 50:11-18.
14. Mohammadin RN, Azarpar E, Moradi M. Effect of different nitrogen and micronutrients fertilizer rates on yield and yield components of rice. World Applied Science Journal. 2011; 13(3):419-423.

15. Park ST. Biological yield and harvest index relation to major cultivation methods in rice plant. Effect of nitrogen application on biological yield and harvest index. Research Reports of the Rural Development Administration, Crops. Kore a Republic. 1987; 29(2):92106.

16. Pramanik K, Bera AK. Effect of seedling age and nitrogen fertilizer on growth, chlorophyll content, yield and economics of hybrid rice (Oryza sativa L.). International Journal of Agronomy and Plant Production. 2013; 4:3489-3499.

17. Prasad RV, Subbaiah G, Chandrasekhar K, Prasuna RP. Validation of nitrogen recommendations for popular rice (Oryza sativa L.) varieties of costal Andhra Pradesh. The Andhra Agricultural Journal. 2011; 58(1):1-4.

18. Prasad M. Biological yield and harvest index of rice. Oryza. 1981; 18(1):31-34.

19. Rao VU, Reddi RY, Radha KC, Raghava RC. Effect of organic and inorganic sources of nitrogen on growth, yield, nitrogen uptake and economics of lowland rice. Madras Agricultural Journa. 2004; 91(7-12):389-393.

20. Shimoyama S. Effects of silicic acid on the lodging tolerance and the alleviation of wind damage to rice plants in the growth stage toward heading. In: Okuda A (ed) Studies on the advancement of yield potentials of crop plants with the adoption of silisic acid inputs. Report Research by Minority Education. 1958; 48:57-59.

21. Sommer MD, Fuzyakov, Breuer J. Silicon pools and fluxes in soils and landscapes-a review. Journal of Plant Nutrition and Soil Science. 2006; 169:310-329.

22. Stalin P, Thiyagarajan TM, Rangarajan R. Nitrogen application strategy and use efficiency in rice. Oryza. 1999; 36(4):322-326.

23. Takahashi T. Uptake mode and physiological functions of silica. Japan journal of soil science and plant nutrition. 1995; 49:357-360.

24. Takahashi E, Ma IF, Miyake Y. The Possibility of silicon as an essential element for higher plants. Comments Agriculture Food Chemistry. 1990; 2:99-122.

25. Yang S, Xie L, Yuan JC. Effect of nitrogen rate and transplanting density on physical and chemical characteristics and lodging resistance of culms in hybrid rice. In Acta Agronomica Sinica. 2009; 35(1):93-103.

26. Wang CY, Dai XL, Shi YH, Wang ZL, Chen XG, He MR et al. Effects of nitrogen application rate and plant density on lodging resistance in winter wheat. Acta

27. Agronomic Sinica. 2012; 38:121-128.

28. Wei FZ, Li JC, Wang CY, Qu HJ, Shen XS. Effects of nitrogenous fertilizer application model on culm lodging resistance in winter wheat. Acta Agronomic Sinica. 2008; 34:1080-1085.

29. Zhang J, Li GH, Song YP, Zhang WJ, Yang CD, Wang $\mathrm{SH}$ et al. Lodging resistance of super-hybrid rice Yliangyou 2 in two ecological regions. Acta Agronomic Sinica. 2013; 39:682-692.

30. Zhaowen MO, Shenggang P, Bingshun L, Hua T, Meiyang D, Qingshan L et al. Growth and yield response of five "super" rice (Oryza sativa L.) varieties to nitrogen fertilizer rates. Journal of Food Agriculture and Environment. 2013; 11(3-4):1257-1262. 